\title{
Projection Profile Matching for Intraoperative MRI Registration Embedded in MR Imaging Sequence
}

\author{
Nobuhiko Hata, Junichi Tokuda, Shigeo Morikawa, and Takeyoshi Dohi \\ Graduate School of Information Science and Technology, The University of Tokyo \\ Molecular Neuroscience Research Center, Shiga University of Medical Science \\ \{noby, junichi,dohi\}@atre.t.u-tokyo.ac.jp \\ morikawa@belle.shiga-med.ac.jp
}

\begin{abstract}
Fast image registration for magnetic resonance image (MRI)-guided surgery using projection profile matching embedded in MR pulse sequence is proposed. The method can perform two-dimensional image registration by matching projection profiles acquired with zero-degree phase encoding. The matching process continuously measures displacement by optimizing cross correlation value from profiles acquired 64 times by a special pulse excitation and echo acquisition in one imaging cycle. A phantom experiment concluded that the method can perform the registration in $25 \mathrm{~ms}$ with the accuracy of $0.50 \mathrm{~mm}$ out of $100 \mathrm{~mm}$ field of view. The paper also includes in-vivo experiment to registration MRI arm in motion. Unlike previously reported image registration by post-processing, the method is suitable in intraoperative setting where fast registration is in great need.
\end{abstract}

\section{Introduction}

The role of intraoperative Magnetic Resonance Image (MRI)-guided surgery becomes increasingly important reflecting the trend of minimally invasive therapy ${ }^{1-3}$. The MRI is suitable for intraoperative use for its oblique imaging capabilities, soft tissue discrimination and the detailed delineation of vascular structures. Additionally, MR imaging has unique potential capabilities for functional, thermal, and physiologic imaging.

Despite its obvious advantage in surgical guidance and monitoring, intraoperative MRI has some limitations in the image quality in comparison to conventional diagnostic high tesla MR, since requirements for interventional use have some impact on intraoperative MR imaging capability. An example of such requirement is the use of surface coils for surgical access, thus causing the inhomogeneous sensitivity in intraoperative images.

One of the solutions to overcome these limitations in intraoperative MRI is to fuse pre-operative MRI and/or other multi-modality images to provide complementary information $^{4-8}$. Fusion of pre-operative image and intraoperative MRI is possible by registering skin markers ${ }^{8,9}$, intensity-based image matching ${ }^{4,7}$, or by taking preoperative MRI scans just after anesthesia, assuming that the patient doesn't move thereafter ${ }^{6,10}$. While these previously published registration methods have indicated the significance of the intra-operative multi-modal registration, they include post- 
processing of intra-operative images or cumbersome marker setting, thus inherently requires a few minutes or more to achieve a registration. Though the other methods ${ }^{5,6}$ do not need procedures for registration, they rely on an assumption that patient or cured lesion does not move throughout the case, which is specific to their targeted clinical applications.

A novel image fusion method proposed in this paper, is to perform fast registration in 10 to $100 \mathrm{~ms}$ order by embedding the image registration process in MRI imaging sequence. The method tries to match the projection profiles taken before and after the motion, along phase and frequency encoding direction, by optimizing the cross correlation. The newly proposed method is significant in engineering perspective since it is, by the authors' knowledge, the first proposal in publication to include image registration in MR image acquisition, thus notably reducing the computing time. The paper is clinically significant since the newly proposed method, when implemented in clinical intraoperative imager, will enable physicians' real-time access to preoperative multi-modal image thus achieving the enhanced and detailed assessment of cured region.

The objective of this paper is to present the principle of profile matching and its ease of inclusion in MR imaging sequence. The method is then evaluated by phantom study to assess the accuracy and performance of the method, and by in-vivo experiments to investigate the clinical feasibility.

\section{Materials and Methods}

\section{MR Projection Profile}

In two-dimensional Fourier encoding, the signal received by Radio Frequency (RF) coil is given by:

$$
S(t)=!_{-\infty}^{\infty} !_{-\infty}^{\infty} M(x, y) \exp \left\{-i\left(\gamma G_{x} x t+\gamma G_{y} y t_{y}\right)\right\} d x d y
$$

where $M(x, y)$ is the distribution of magnetization, $\gamma$ is the gyromagnetic ratio, and $G_{x}, G_{y}$ is the magnetic field gradient. Consider the case that the RF coil receives echo without phase encoding $\left(G_{y}=0\right)$. The received signal is

$$
S(t)=!_{-\infty}^{\infty} !_{-\infty}^{\infty} M(x, y) \exp \left(-i \gamma G_{x} x t\right) d x d y
$$

For notational convenience, we can rewrite this equation as

$$
S\left(k_{x}\right)=!_{-\infty}^{\infty} !_{-\infty}^{\infty} M(x, y) \exp \left(-i k_{x} x\right) d x d y
$$

where $k_{x}=\gamma G_{x} t$. By applying Fourier Translation to equation (3), we obtain the projection profile along $\mathrm{x}$-axis.

$$
p(x)=\frac{1}{2 \pi} !_{-\infty}^{\infty} S\left(k_{x}\right) d k_{x}=!_{-\infty}^{\infty} M(x, y) d y
$$

Similarly, the projection onto y-axis can be obtained by taking echo without phase encoding $\left(G_{x}=0\right)$. 


\section{Profile Matching by Cross Correlation}

Under the condition of rigid body motion, the two-dimensional translation of the subject causes the 1-dimensional translation of projection profiles along each axis. The shape of projection profile is not deformed by the translation. This means that the displacement of the subject can be quantified by matching projections before and after the motion. Now we are given two projection profiles: $p_{0}(x)$ and $p_{n}(x) \cdot p_{0}(x)$ is the baseline projection profile along $\mathrm{x}$-axis, and $p_{n}(x)$ is the $n^{\text {th }}$ projection profile along $\mathrm{x}$-axis. Estimating the x-directional translation of the subject between the baseline and $n^{\text {th }}$ profile acquisition at $\Delta x, p_{n}(x)$ is almost identical to $p_{0}(x+\Delta x)$ when the parameter $\Delta x$ is actual value. Thus we can determine the translation parameter $\Delta x$ by maximizing the similarity measure between $p_{n}(x)$ and $p_{0}(x+\Delta x)$. We used cross correlation for this similarity measure. The problem is denoted as follows:

$$
\Delta x=\arg \max \left(C\left(p_{0}(x+\Delta x), p_{n}(x)\right)\right)
$$

where $C\left(p_{0}(x+\Delta x), p_{n}(x)\right)$ is cross correlation between $p_{0}(x+\Delta x)$ and $p_{n}(x)$. The method can be applied to the other direction by exchanging the direction of phase encoding and frequency encoding.

\section{Experiments}

The method was evaluated by two set of experiments: first, the combined MR imaging sequence for imaging and matching is implemented in 2.0T experimental MRI scanner to compare the matching results with gold standard measured by external displacement sensor. In the second set of experiments, an arm was tracked by the newly proposed registration method to assess the feasibility of the method in clinical setting.

In the phantom feasibility study, the goal of the experiment was to evaluate the accuracy of the proposed method in a 2.0 Tesla experimental scanner (CSI Omega System, Bruker, Fremont, CA). The specially designed placement stage is installed on the scanner's bore. The movement of the stage was restricted to only frequencyencoded direction for imaging. The moving phantom was fixed on the stage (Fig. 2).

The pulse sequence was modified gradient echo (TR/TE: $40 \mathrm{~ms} / 7 \mathrm{~ms}$, field of view (FOV) $100 \mathrm{~mm}$, matrix 256x128), which is near the sequence of semi-real time imaging used in an intraoperative MRI. In this pulse sequence, non-phase-encoded pulse excitation and echo acquisition process was inserted every other echo acquisition for imaging. Total of 64 projection profiles per each axis were obtained during one imaging (approx. 10 seconds). The stage was moved manually up to 30-40 $\mathrm{mm}$ while scanning, and the stage was tracked by Charge-Coupled Device (CCD) laser displacement sensor (LK-500, Keyence, Osaka, Japan) with resolution of $50 \mu \mathrm{m}$ and sampling cycle of $1024 \mu \mathrm{s}$. Both scanning and laser displacement sensor were synchronized by a shared pulse generator.

We also performed in-vivo study by imaging axial images of an arm of a volunteer (male, 22-y-o). The arm was continuously moved along horizontal and vertical di- 
rection in the scanner's bore at approximately 20 to $30 \mathrm{~mm}$ per second. Imaging sequence and the scanner were same as those used in phantom experimental study. In both phantom and in-vivo experiments, projection matching process was executed retrospectively in a workstation (SunBlade1000, Sun Microsystems, Palo Alto, CA) after all data acquisition had been completed. All matching program is developed on MATLAB (The MathWorks, Natick, MA).
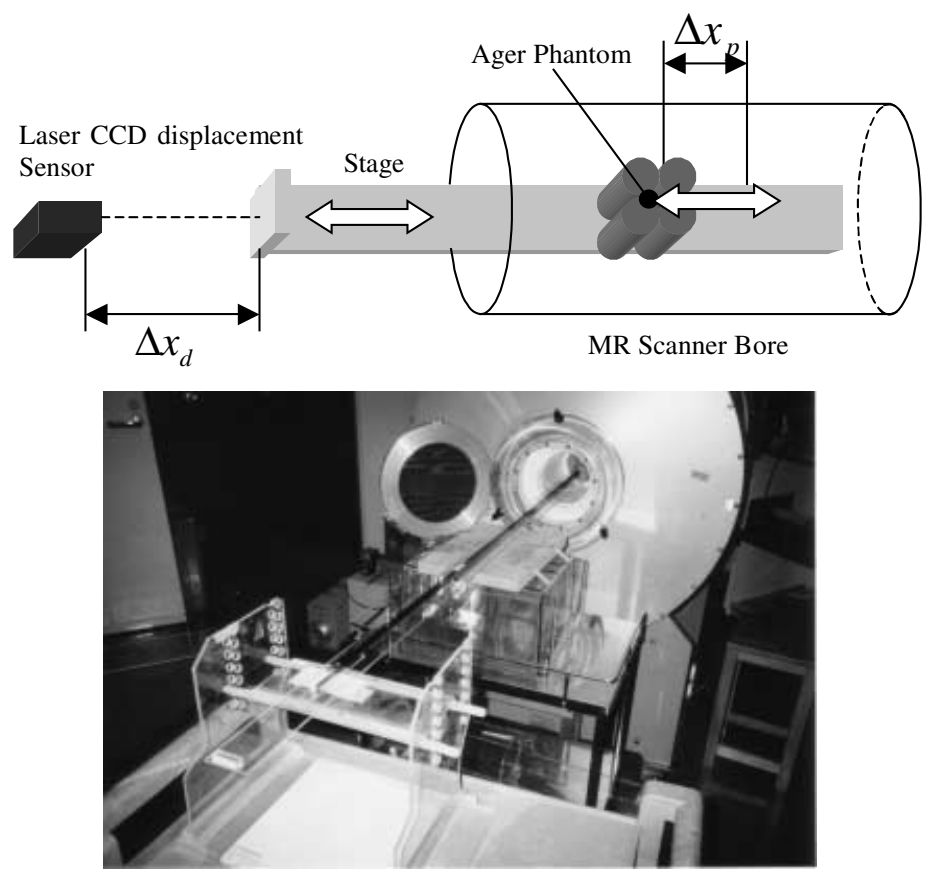

Fig. 1. A schematic presentation of configuration of phantom experiment (top) and the experimental setup in 2.0T experimental MR scanner (bottom). The stage was tracked by the laser CCD displacement sensor to measure actual displacement of the age phantom, $\Delta x_{d}$, and compared to $\Delta x_{p}$ computed by the proposed method

\section{Results}

Eighteen images (64 projection profiles per image for each direction) were acquired for each experiment and 1140 were successfully completed. The standard deviation of the error between the value of the phantom displacement computed from projection data and the value measured by the CCD laser displacement sensor was $0.3 \mathrm{~mm}$ out of $100 \mathrm{~m}$ FOV. Optimization of each projection profile was completed in $36 \mathrm{~ms}$ (CPU time) in average.

From the in-vivo arm study, we could confirm the re-alignment of the displaced arm in the original position. Figure 2 illustrates an illustrative example from the experiment. 

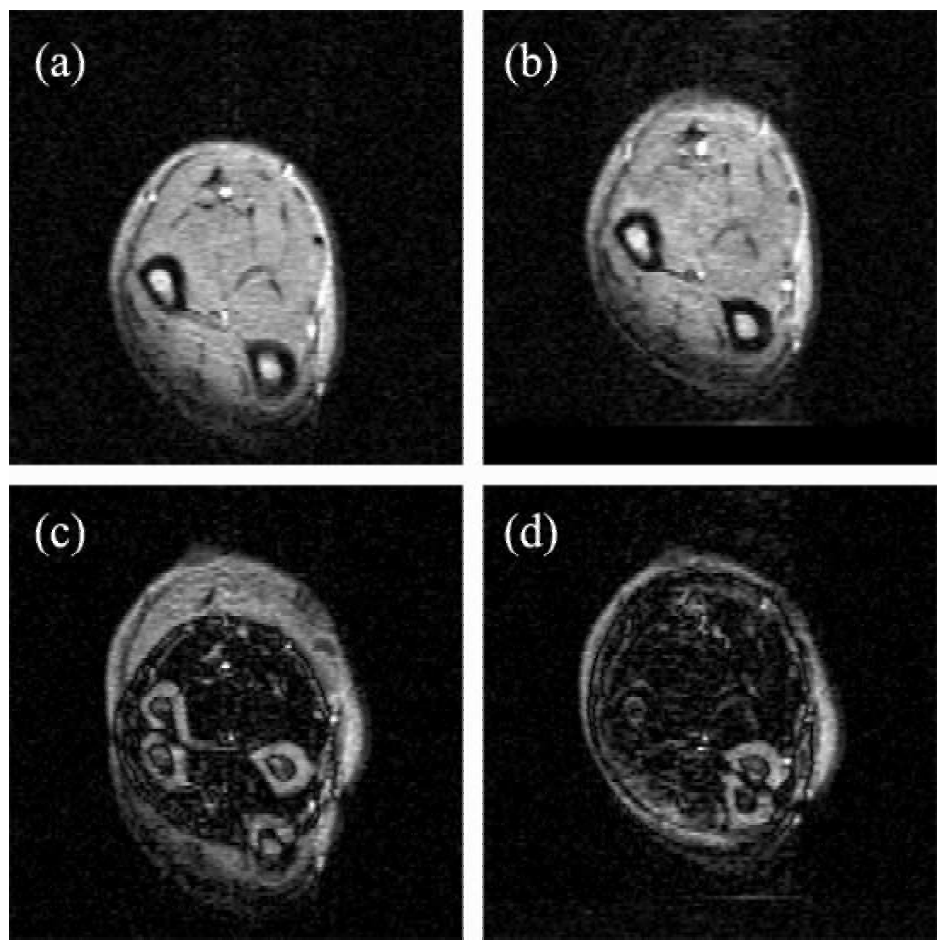

Fig. 2. The results from the in-vivo arm scan using 2.0T experimental MRI scanner. (a) is an illustrative example of non-registered image, and (b) shows the image (a) registered to the reference image. (c) and (d) are the subtraction of the image (a) to the reference image, and (b) to the reference image, respectively. Note that the registered image (b) well matches the baseline image generating less mismatched area in the subtraction image (d)

\section{Discussions}

The objective results from the phantom image were satisfactory, especially the computing time $36 \mathrm{~ms}$ per one profile matching. This indicates, if the matching and image acquisition can be performed in parallel and TR value in MR imaging is small enough, the matching requires nominal additional time to normal imaging.

Our experience, not presented in this paper, indicates that the accuracy of matching is affected by signal-to-noise ratio (SNR). The accuracy is also affected by inhomogeneity of coil sensibility. Since coil sensitivity is highest in the center of the coil, the larger the displacement of phantom becomes, the weaker the signal from phantom is. The matching error reported in this paper $(0.3 \mathrm{~mm}$ out of $100 \mathrm{mmFOV})$ is presumable smaller than the number we can expect in clinical small tesla intraoperative MRI scanner.

Our method is based on the assumption that the subject is rigid, and not deformable. Therefore, the current implementation of the method does not function well when a part of anatomical structure in an image moves, while the rest is still. A pos- 
sible modification to the method to solve this issue is the segmenting out the moving anatomy from a baseline image and takes profiles from the segmented part. The projection profiles of the moving object during scans were calculated by subtracting the baseline profile of the static object from each projection profiles.

\section{Conclusion}

We have demonstrated the method for fast registration embedded in MR pulse sequence. The method successfully performed on phantom study with enough accuracy and speed. This method has capability to apply to 3-dimensional Fast Fourier Transformation (FFT) imaging, and estimate the 3-dimensional motion of the subject.

\section{Acknowledgements}

This study was funded by Grant-in-Aid Scientifi Research (A-14702070 and B13558103). NH was supported by Suzuken Memorial Foundation, Kurata Grants, and Toyota Physical \& Chemical Research Institute.

\section{References}

1. Ladd, M. E., Quick, H. H. \& Debatin, J. F. Interventional MRA and intravascular imaging. J Magn Reson Imaging 12, 534-46. (2000).

2. Quesson, B., de Zwart, J. A. \& Moonen, C. T. Magnetic resonance temperature imaging for guidance of thermotherapy. J Magn Reson Imaging 12, 525-33. (2000).

3. Lewin, J. S., Metzger, A. \& Selman, W. R. Intraoperative magnetic resonance image guidance in neurosurgery. J Magn Reson Imaging 12, 512-24. (2000).

4. Gering, D. T. et al. An integrated visualization system for surgical planning and guidance using image fusion and an open MR. J Magn Reson Imaging 13, 967-75. (2001).

5. Hata, N. et al. MR imaging-guided prostate biopsy with surgical navigation software: device validation and feasibility. Radiology 220, 263-8. (2001).

6. Nabavi, A. et al. Serial intraoperative magnetic resonance imaging of brain shift. Neurosurgery 48, 787-97; discussion 797-8. (2001).

7. Roche, A., Pennec, X., Malandain, G. \& Ayache, N. Rigid registration of 3-D ultrasound with MR images: a new approach combining intensity and gradient information. IEEE Trans Med Imaging 20, 1038-49. (2001).

8. Samset, E. \& Hirschberg, H. Neuronavigation in intraoperative MRI. Comput Aided Surg 4, 200-7 (1999).

9. Flask, C. et al. A method for fast 3D tracking using tuned fiducial markers and a limited projection reconstruction FISP (LPR-FISP) sequence. J Magn Reson Imaging 14, 617-27. (2001).

10. Jolesz, F. A., Nabavi, A. \& Kikinis, R. Integration of interventional MRI with computerassisted surgery. J Magn Reson Imaging 13, 69-77. (2001). 